\title{
Mathematical Model on Reliability of Variation Process of Rolling Bearing Vibration Performance
}

\author{
Xintao Xia ${ }^{\mathrm{a}}$, Liang $\mathrm{Ye}^{\mathrm{b}^{*}}$ and Zhen Chang ${ }^{\mathrm{c}}$ \\ School of Mechatronical Engineering, Henan University of Science and Technology, Luoyang 471003, \\ China \\ axiaxt1957@163.com, b172682823@qq.com, zhenc1992@163.com
}

Keywords: Rolling bearings; Reliability; Variation process; Maximum entropy principle; Grey bootstrap principle

\begin{abstract}
A reliability evaluation model is created to analyze the variation process of time series based on the maximum entropy and grey bootstrap principles. The original sample data is collected by measuring the vibration acceleration of time series. Then the simulated vibration acceleration data is obtained using Monte Carlo method for different wear diameters. According to the maximum entropy principle, the estimated true value and confidence interval can be calculated for the reliability parameter of the intrinsic sequence. The grey bootstrap principle is applied to obtain a large number of sample data by resampling from the parametric sample data. The variation frequencies of time series can be achieved using Poisson counting principle. The estimated true value function and the upper and lower bound functions of reliability are achieved based on the variation frequencies. The variation probabilities can be calculated for different time series. The reliability evaluation model is proven to be able to be used to take intervention measures before the vibration performance fails, which is under the condition that the possibility distribution is unknown for sample data.
\end{abstract}

\section{Introduction}

Rolling bearing is one of the most commonly used mechanical equipment, which is an important supporting of shaft and other rotating components. Whether its performance is good or bad plays a very important role for the normal operation of the equipment. The prediction on performance reliability variation (degradation) process of rolling bearings is to predict the failure probability and performance reliability of rolling bearings according to the current performance data [1]. Then the variation process of bearing performance is studied to find the risks that the vibration performance will vary, so nasty accident will be avoided. Therefore, it is of great academic and application value to study the performance variation process of rolling bearings.

The available performance reliability prediction methods generally assume that the probability density functions and the performance failure thresholds of samples are known, so the performance reliability can be calculated and analyzed. For example, Ali proposed a method based on the data-driven prognostic approach and explored the combination of Simplified Fuzzy Adaptive Resonance Theory Map (SFAM) neural network under the condition of Weibull distribution [2]. Xia raised the bootstrap weighted-norm method and assumed that the life of rolling bearings is in accordance with three-parameter Weibull distribution to evaluate the optimum confidence interval of reliability [3]. Jiang put forward the modified MLE (MMLE) for estimating the parameters based on the zero-failure data and assumed that product lifetime followed a Weibull distribution [4]. Dan transformed the Weibull distribution into exponential distribution by using rolling bearing zero-failure data to calculate the characteristic life estimation values of Weibull distribution [5]. However, some probability distribution information on performance degradation is unknown. For example, the failure probability distributions of the vibration and noise, friction torque and fatigue life performances are still unknown or uncertain. Xia fused the grey bootstrap 
principle into Poisson process and proposed the grey bootstrap Poisson method to forecast the vibration performance reliability variation process of rolling bearing with an unknown probability distribution [6].

For sample data with unknown probability distributions, it is difficult in using available methods to solve the reliability assessment problems. So far, it has been an important scientific and technical problem. In view of this, this paper fuses the maximum entropy principle $[7,8,9]$ into the grey bootstrap principle $[10,11]$ and the model for evaluating the variation process of vibration performance reliability of rolling bearings is proposed under the condition without any probability distribution or prior information on sample data. The evaluation model can be used to take intervention measures before vibration performance of bearings fails to avoid the occurrence of serious accidents.

\section{Mathematical Model}

The time sequences are obtained by periodic sampling of vibration acceleration of rolling bearings. The intrinsic sequence is marked as the first time series and expressed by the vector $\boldsymbol{X}_{0}$ as follows:

$$
\boldsymbol{X}_{0}=\left(x_{1}, x_{2}, \ldots, x_{k}, \ldots, x_{N}\right)
$$

where $x_{k}$ is $k$ th performance data, $k=1,2,3, \ldots, N(N \geq 1000) ; N$ is the number of performance data in the intrinsic sequence.

The $n$th time series is divided into $D$ sequences, and the sample data of the $d$ th sub sequence is expressed by vector $\boldsymbol{X}_{n d}$.

$$
\boldsymbol{X}_{n d}=\left(x_{n d}(1), x_{n d}(2), \ldots, x_{n d}(l), \ldots, x_{n d}(L)\right)
$$

where $x_{n d}$ is the sample data of the $d$ th sub sequence of the $n$th time series; $d$ is the sequence number of sub series. $d=1,2,3, \ldots, D ; x_{n d}(l)$ is the $l$ th data of the $d$ th sub sequence of the $n$th time sequence; $l$ is the sequence number of performance data in the sub sequence, $l=1,2,3, \ldots, L ; L$ is the number of sample data in the sub sequence, $L \times D \geq 1000, D \leq 10$.

Maximum Entropy Principle. According to the maximum entropy principle, the probability density functions should meet the condition that the entropy value is the maximum.

$$
H=-\int_{\Omega} f(x) \ln f(x) \mathrm{d} x \rightarrow \max
$$

where $H$ is the information entropy; $\Omega$ is the feasible region for random variable $x, f(x)$ is the probability density function of continuous variable $x$.

Eq. (3) should meet the constraint conditions as follows:

$$
\int_{\Omega} x^{i} f(x) \mathrm{d} x=m_{i} ; i=0,1, \ldots, m ; m_{0}=1
$$

where $i$ is the order of origin moment; $m_{i}$ is the $i$ th origin moment; $m$ is the order of the highest origin moment.

All origin moments of the performance data in the intrinsic sequence are given by

$$
m_{i}=\frac{1}{N} \sum_{k=1}^{N}\left(x_{k}\right)^{i}
$$

where $k$ is the sequence number of performance data, $k=1,2,3, \ldots, N(N \geq 1000) ; N$ is the number of performance data of the intrinsic sequence; $i$ is the order of origin moment.

The probability density function $f(x)$ can be expressed based on Lagrange multiplier method as follows:

$$
f(x)=\exp \left(\sum_{i=0}^{m} c_{i} x^{i}\right)
$$


where $c_{i}$ is the $i$ th Lagrange multiplier; $i$ is the order of origin moment, $i=0,1, \ldots, m ; m$ is the order of the number of highest order of origin moment.

The first multiplier $c_{0}$ can be given by

$$
c_{0}=-\ln \left(\int_{\Omega} \exp \left(\sum_{i=1}^{m} c_{i} x^{i}\right) \mathrm{d} x\right)
$$

Other $m$ multipliers should meet the condition as follows:

$$
m_{i} \int_{\Omega} \exp \left(c_{i} x^{i}\right) \mathrm{d} x-\int_{\Omega} x^{i} \exp \left(\sum_{j=1}^{m} c_{j} x^{j}\right) \mathrm{d} x=0 ; i=1,2, \ldots, m
$$

Set a significant level, $\alpha \in[0,1]$, the maximum entropy estimated interval of continuous variable $x$ can be calculated as follows:

$$
\left[x_{\mathrm{L}}, x_{\mathrm{U}}\right]=\left[x_{\frac{\alpha}{2}}, x_{1-\frac{\alpha}{2}}\right]
$$

where $x_{\mathrm{L}}$ is the lower boundary value of estimated interval; $x_{\mathrm{U}}$ is the upper boundary value of estimated interval.

The estimated true value $x_{0}$ of performance parameter of time series is given by

$$
x_{0}=\int_{\Omega} x f(x) \mathrm{d} x
$$

The maximum entropy estimation interval $\left[x_{\mathrm{L} 0}, x_{\mathrm{U} 0}\right]$ of the intrinsic sequence is calculated, where $x_{\mathrm{L} 0}$ is the lower bound value of the estimated interval and $x_{\mathrm{U} 0}$ is the upper bound value of estimated interval.

Prediction on the Variation Probability. Count the number $N_{n d}$ that performance data of the $d$ th sub sequence of the $n$th time series falls outside the estimated interval $\left[x_{\mathrm{L} 0}, x_{\mathrm{U} 0}\right]$. Based on the Poisson counting method, the frequencies that performance data falls outside the estimated interval can be given by

$$
\lambda_{n d}=\frac{N_{n d}}{L}
$$

Grey Bootstrap Principle. The grey bootstrap principle fuses the bootstrap principle into grey prediction principle GM $(1,1)$. A data sequence vector $\lambda$ can be given by

$$
\lambda=\left\{\lambda_{n 1}, \lambda_{n 2}, \ldots, \lambda_{n D} ; n=2,3, \ldots, r\right\}
$$

$B$ bootstrap resampling samples can be expressed by vector $\boldsymbol{Y}_{\text {Bootstrap }}$ as follows:

$$
\boldsymbol{Y}_{\text {Bootstrap }}=\left(\boldsymbol{Y}_{1}, \boldsymbol{Y}_{2}, \ldots, \boldsymbol{Y}_{b}, \ldots, \boldsymbol{Y}_{B}\right)
$$

where $\boldsymbol{Y}_{b}$ is the $b$ th sample; $b$ is the order number of sample, $b=1,2, \ldots, B$.

$$
\boldsymbol{Y}_{b}=\left\{y_{b}(u)\right\}
$$

where $y_{b}(u)$ is the $u$ th re-sampling sample of $\boldsymbol{Y}_{b} ; u=1,2, \ldots, D$.

According to the grey prediction principle GM $(1,1)$, the predicted value $\hat{y}_{b}(w)$ can be given by

$\hat{y}_{b}(w)=\lambda_{b}(w)-\lambda_{b}(w-1) ; w=T+1$

where $w$ and $T$ are certain moments in the future.

Sequence vector $\hat{\lambda}_{w}$ can be obtained with $B$ data as follows:

$$
\hat{\lambda}_{w}=\left\{\hat{y}_{b}(w)\right\} ; b=1,2, \cdots, B ; w=T+1
$$


The Estimation of Reliability Functions.The estimated true value of reliability function can be expressed by $R_{n}(t)$ as follows:

$R_{n 0}(t)=\exp \left(-\lambda_{n 0} t\right)$

where $\lambda_{n 0}$ is the estimated true value of variation frequency of the $n$th sequence; $n=1,2, \ldots, r$.

The upper bound reliability function can be given by with $R_{n \mathrm{U}}(t)$ :

$R_{n \mathrm{U}}(t)=\exp \left(-\lambda_{n \mathrm{U}} t\right)$

where $\lambda_{n \mathrm{U}}$ is the upper bound value of variation frequency of the $n$th sequence.

The lower bound reliability function can be expressed by $R_{n \mathrm{~L}}(t)$ as follows:

$R_{n \mathrm{~L}}(t)=\exp \left(-\lambda_{n \mathrm{~L}} t\right)$

where $\lambda_{n \mathrm{~L}}$ is the lower bound value of variation frequency of the $n$th sequence.

The Estimation of Variation Probabilities. The variation probabilities $P_{n}(t)$ of the time series are given by

$$
P_{n}(t)=1-\left(\int_{t=0}^{t_{n}} f_{1}(t) \mathrm{d} t+\int_{t=t_{n}}^{+\infty} f_{n}(t) \mathrm{d} t\right)
$$

where $t$ is the time when bearings operate; $t_{n}$ is the moment when the variant probability density functions of the $n$th sub sequence and the intrinsic sequence intersect, $f_{l}(t)$ and $f_{n}(t)$ are the variant probability density functions of the intrinsic sequence and the $n$th sub sequence, $n=2,3, \ldots, r$.

$$
\begin{aligned}
& f_{n}(t)=\frac{\mathrm{d}\left(1-R_{n}(t)\right)}{\mathrm{d} t}=\lambda_{n} \exp \left(-\lambda_{n} t\right) \\
& f_{1}(t)=\lambda_{1} \exp \left(-\lambda_{1} t\right)
\end{aligned}
$$

\section{Summary}

According to the maximum entropy and grey bootstrap principles, a model is established to evaluate the variation process of vibration performance reliability of rolling bearings, which can be applied to calculate the estimated true value function and the reliability upper and lower bound functions. The reliability variation frequencies and variation probabilities can be obtained for different time series to study the variation process under different wear diameters.

For sample data with unknown probability distributions, the reliability evaluation model is proven to be scientific and reasonable in solving the reliability variation probability problems.

\section{Acknowledgements}

This project was funded by the National Natural Science Foundation of China (Grant Nos. 51475144 and 50675011).

\section{References}

[1] X.T. Xia, B.M. Zhang and Y.Z. Xu. Variation Process Evaluation for Performance and Reliability of Rolling Bearing Using Poor Information Theory, first ed., Science Press, Beijing, 2013.

[2] Jaouher Ben Ali, Brigitte Chebel-Morello and Lotfi Saidi, et al. Accurate bearing remaining useful life prediction based on Weibull distribution and artificial neural network. Mechanical Systems and Signal Processing, 2015, (56-57): 150-172. 
[3] X.T. Xia, Y.Z. Xu and Y.P. Jin. Assessment of optimum confidence interval of reliability with three-parameter Weibull distribution using bootstrap weighted-norm method. Journal of Aerospace Power, 2013, 28(3): 481-488.

[4] P. Jiang, J.H. Lim and M.J. Zuo, et al. Reliability estimation in a Weibull lifetime distribution with zero-failure field data. Quality and Reliability Engineering International, 2010, 26(7): 691-701.

[5] S.J. Dan, H.L. Lou and X.L. Li, et al. Reliability estimation for rolling bearings based on zero-failure data. Bearing, 2013, (9): 22-24.

[6] X.T. Xia, Y.Y. Meng and Ming Qiu, et al. Forecasting for variation process of reliability of rolling bearing vibration performance using grey bootstrap Poisson method. Journal of Mechanical Engineering, 2015, 51(9): 97-103.

[7] X.F. Dong, H.L. Li and H.J. Yu. Degradation analysis of machine tool spindle based on maximum entropy and discrimination information. Journal of Virration and Shock, 2013, 32(5): 62-64.

[8] L. Ye, Y. Zhu and Y.N. Zhao, et al. An improved approach based on maximum entropy principle for wind resource distribution characteristics. Proceedings of the CSEE, 2014, 34(34): 6093-6099.

[9] J.Z. Zhao, T.X. Xu and H.J. Li, et al. Demand forecasting of missile equipment spare parts based on maximum entropy principle and simulation. Modern Defence Technology, 2014, 42(4): 148-153.

[10] L.Y. Ge, W. Zhao and Z.F. Xu, et al. Error predicting for dynamic measurement of poor information based on grey bootstrap method. Chinese Journal of Aagricultural Machinery, 2011, 42(7): 210-214.

[11] Y. Lu, B.J. Shi and H. Guo, et al. Grey bootstrap evaluation for vibration of deep groove ball bearings. Bearing, 2015, (1): 37-40. 